\title{
Oxygen interaction with the Pd(112) surface: From chemisorption to bulk oxide formation
}

\author{
Alina Vlad,,${ }^{1,2}$ Andreas Stierle, ${ }^{3,2, *}$ Rasmus Westerström, ${ }^{4, \dagger}$ Sara Blomberg, ${ }^{4}$ Anders Mikkelsen, ${ }^{4}$ and Edvin Lundgren ${ }^{4}$ \\ ${ }^{1}$ Synchrotron SOLEIL, Saint Aubin BP 48, F-91192 Gif-sur-Yvette Cedex, France \\ ${ }^{2}$ Max-Planck Institut für Intelligente Systeme (formerly Max-Planck Institut für Metallforschung), D-70569 Stuttgart, Germany \\ ${ }^{3}$ Universität Siegen, D-57072 Siegen, Germany \\ ${ }^{4}$ Division of Synchrotron Radiation Research, Institute of Physics, University of Lund, SE-221 00 Lund, Sweden
}

(Received 20 February 2012; revised manuscript received 15 May 2012; published 5 July 2012)

\begin{abstract}
We investigated the interaction of oxygen with the $\mathrm{Pd}(112)$ surface from ultrahigh vacuum up to 5 mbars oxygen partial pressure in a temperature range from 523 to $673 \mathrm{~K}$. We combined in situ surface x-ray diffraction with scanning tunneling microscopy, high-resolution core-level spectroscopy, and low-energy electron diffraction. A structural model of the clean $\operatorname{Pd}(112)$ is proposed based on the x-ray-diffraction data. The morphology of the $\operatorname{Pd}(112)$ surface is strongly influenced by the oxidation conditions: at $673 \mathrm{~K}$, upon exposure to oxygen at pressures from $2 \times 10^{-8}$ to $5 \times 10^{-5}$ mbar, the (112) surface undergoes a massive rearrangement and (113)and (335)-type facets are formed. Further increase of the $\mathrm{O}_{2}$ partial pressure leads to a new rearrangement into (111)- and (113)-type facets. This is in contrast to the previous observation that (112) facets are stabilized on $\mathrm{MgO}$ supported Pd nanoparticles under oxygen exposure [P. Nolte, A. Stierle, N. Kasper, N. Y. Jin-Phillipp, N. Jeutter, and H. Dosch, Nano Lett. 11, 4697 (2011)]. Based on the core-level spectroscopy and scanning tunneling microscopy measurements, the transition from chemisorbed oxygen to surface oxide formation was identified to take place at pressures of $10^{-3} \mathrm{mbar}_{2}$ and $623 \mathrm{~K}$. Kinetic barriers for the formation of the PdO bulk oxide are observed to be reduced compared to low index Pd surfaces.
\end{abstract}

DOI: 10.1103/PhysRevB.86.035407

PACS number(s): 68.35.B-, 61.05.cf, 68.37.Ef

\section{INTRODUCTION}

Palladium is an important material as an oxidation catalyst for gas exhaust cleaning or during hydrogenation. ${ }^{1}$ The interaction of oxygen with $\mathrm{Pd}$ is involved in various chemical reactions, which motivated oxidation studies of low index $\mathrm{Pd}$ single-crystal surfaces. ${ }^{2-4}$ In a real catalyst, the material is, however, present in nanoparticular form, which makes it often difficult to extrapolate from single-crystal surface results. One way to overcome the increased complexity of nanomaterials is the investigation of epitaxial metal nanoparticles with welldefined shape on suitable oxide supports. ${ }^{5}$ Recently, we have demonstrated that during the oxidation of Pd nanoparticles on $\mathrm{MgO}(100)$ new facets with (112) orientation are formed, which is a reversible process under $\mathrm{CO}$ exposure. ${ }^{6}$ The question arises, if the oxygen induced stabilization holds also for extended (112) facets on a vicinal Pd(112) single-crystal surface or if it is a truly nanoscopic effect. As a step back in complexity as compared to nanoparticles, vicinal surfaces allow the investigation of step edges with well-defined adsorption geometry present in a high density on the surface. ${ }^{7-9}$

The present study focuses on the interaction of oxygen with the $\operatorname{Pd}(112)$ surface to gain more insight into the stability of the (112) step geometry under oxygen exposure. In situ surface $\mathrm{x}$-ray-diffraction (SXRD) measurements were performed at various oxygen pressures ranging from ultrahigh vacuum (UHV) to 5 mbars and temperatures between 523 and $673 \mathrm{~K}$. Additional information was obtained by low-energy electron diffraction (LEED), scanning tunneling microscopy (STM) measurements, and high-resolution corelevel spectroscopy (HRCLS). Our results demonstrate that the interaction of oxygen with the $\operatorname{Pd}(112)$ surface gives rise to significant refaceting in different orientations at variance to the observed stability of (112) facets on oxygen exposed Pd nanoparticles.

\section{EXPERIMENTAL DETAILS}

The initial cleaning process of the surface by cycles of $\mathrm{Ar}^{+}$ion sputtering at $1000 \mathrm{eV}$ and annealing to $1000 \mathrm{~K}$ was performed in a stationary ultrahigh vacuum system equipped with an apparatus for low-energy electron diffraction and Auger electron spectroscopy. The in situ oxidation experiments were carried out in a portable UHV high-pressure compatible $\mathrm{X}$-ray-diffraction chamber equipped with an oxidation resistant heater, a sputter gun for ion bombardment of the surface, and gas inlets for oxygen and argon.

The measurements were conducted at the Max Planck Institut für Metallforschung beamline ${ }^{10}$ at the Angströmquelle Karlsruhe using a photon energy of $10 \mathrm{keV}$. The sample temperature was controlled by a Chromel-Alumel type $K$ thermocouple spot welded to its side (temperature accuracy $\pm 10 \mathrm{~K})$. The oxygen pressure was determined by two capacitive gauges $\left(10^{-3}-1\right.$ mbar and $1-1000$ mbar $)$ and by a cold cathode gauge at lower pressures. Above $5 \times 10^{-3}$ mbar the chamber was isolated from the turbomolecular pump and the experiments were performed under static pressure conditions.

A complete structural analysis of the clean surface was performed by measuring crystal truncation rods (CTRs). After background subtraction and application of standard correction factors ${ }^{11}$ a fit of the data was carried out using the software package ROD. ${ }^{12}$ The $\mathrm{x}$-ray-diffraction data presented in this paper are indexed according to the surface unit cell of $\operatorname{Pd}(112)$ (if not otherwise stated) described by the following parameters: $a=6.737 \AA, b=2.75 \AA$, and $c=4.764 \AA$ along the $[1,1, \overline{1}]$, $[1, \overline{1}, 0]$, and $[1,1,2]$ bulk directions, respectively, and $\alpha=\beta=$ $\gamma=90^{\circ}$.

HRCLS and LEED measurements were performed at the beamline I311 at MAX-lab (Lund, Sweden). ${ }^{13}$ The sample was mounted on a tungsten wire, through which it could be heated 
(a) [010]

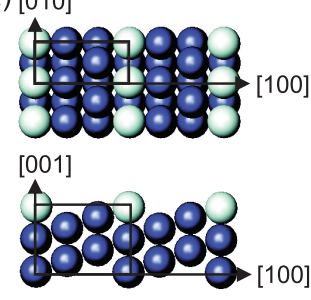

(b)
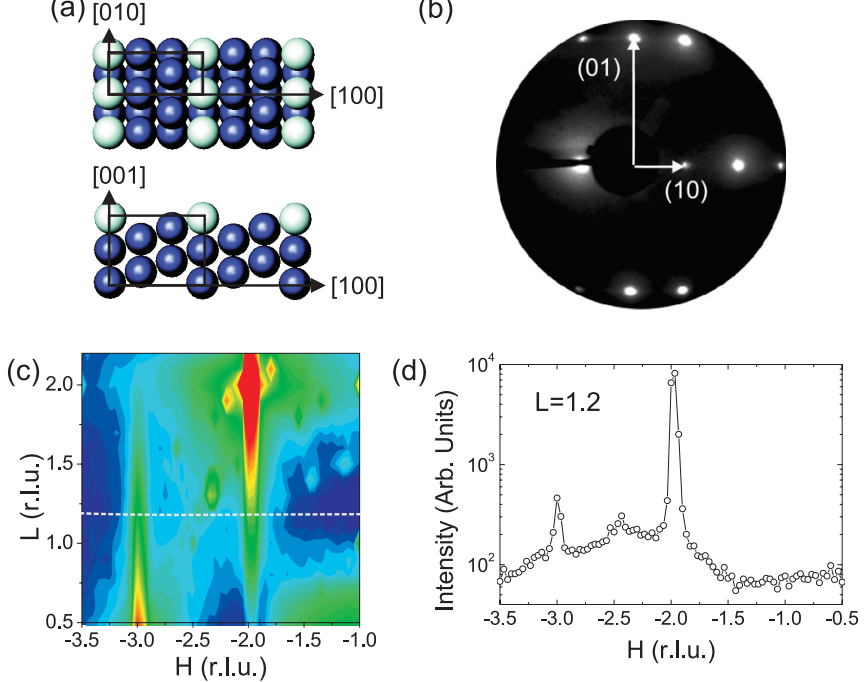

FIG. 1. (Color online) (a) Schematic top and side view of the $\operatorname{Pd}(112)$ surface. The black rectangle marks the (112) surface unit cell used for indexing the $\mathrm{x}$-ray data. The step atoms are represented by the light (blue) spheres. $(b)(1 \times 1)$ LEED pattern from the clean $\operatorname{Pd}(112)$ surface measured at an incident electron energy of $120 \mathrm{eV}$. (c) Experimental map of the diffracted intensity in the $(H, 0, L)$ plane measured for the clean Pd(112) surface at $673 \mathrm{~K}$ in UHV. (d) $H$ scan at $L=1.2$ and $K=0$ from the clean $\operatorname{Pd}(112)$ surface. The signal at $H \approx-2.4$ stems from some residual (111) facets.

to high temperatures by applying an electric current. The temperature of the sample was measured by a Chromel-Alumel thermocouple spot welded on the back side of the crystal. The sample was cleaned by repeated cycles of sputtering with $\mathrm{Ar}^{+}$ ions and annealing up to $1100 \mathrm{~K}$. The surface cleanliness was checked by LEED and x-ray photoemission spectroscopy. The spectra shown here were measured at normal emission using a photon energy of 400 and $620 \mathrm{eV}$ for the $\mathrm{Pd} 3 d^{5 / 2}$ and $\mathrm{O} 1 s$ level, respectively.

STM and LEED images were obtained at the University of Lund using a microscope positioned inside an ultrahigh vacuum system with a base pressure of $1 \times 10^{-10} \mathrm{mbar}$. The STM measurements were performed in constant current mode using electrochemically etched tungsten tips. The sample was cleaned as was done for the HRCLS measurements and exposed to oxygen under similar pressure and temperature conditions as used for the SXRD, LEED, and HRCLS experiments.

For the comparison between the STM, LEED, and HRCLS results with the in situ SXRD measurements at elevated temperatures, it has to be kept in mind that with STM, HRCLS, and LEED a frozen in state of the surface was investigated, whereas the SXRD results reflect more an equilibrium situation.

\section{RESULTS}

\section{A. The clean surface}

The clean surface was investigated by surface $\mathrm{x}$-ray diffraction (SXRD) and low-energy electron diffraction (LEED). Figure 1(a) shows a schematic top and side view of the ideal $\operatorname{Pd}(112)$ surface, which consists of three-atoms-wide (111) terraces separated by monoatomic $\{100\}$ steps, exhibiting threefold (on the terraces and at the step edges) and fourfold

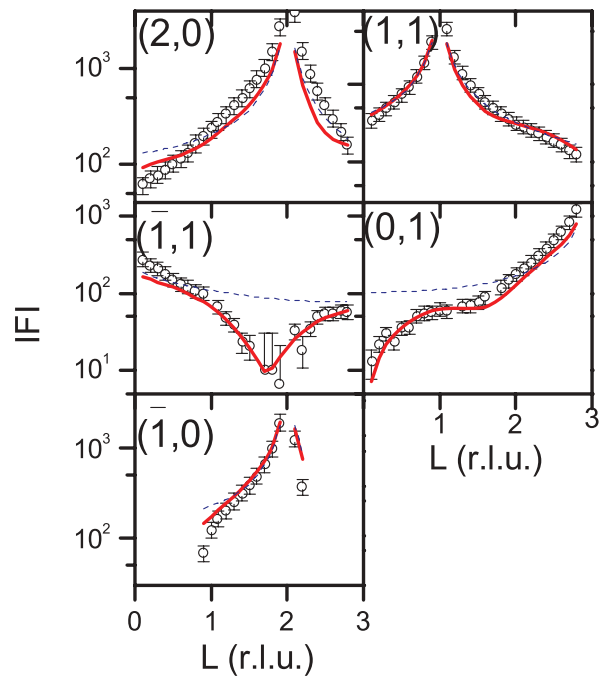

FIG. 2. (Color online) Magnitude of the CTR structure factors of the clean $\mathrm{Pd}(112)$ surface recorded at $673 \mathrm{~K}$ (open circles, together with error bars). The solid (red) line is the best-fit structure factor, as described in the text. The structure factors calculated for an ideal $\operatorname{Pd}(112)$ surface are shown for comparison by the dashed (blue) line.

(at the step edges only) adsorption sites. The LEED pattern of the clean surface at room temperature is shown in Fig. 1(b), and it indicates a well-ordered, unreconstructed surface, in agreement with Ref. 14. Figure 1(c) shows an experimental reciprocal space map of the diffracted intensity in the $(H, 0, L)$ plane obtained for the clean $\operatorname{Pd}(112)$ surface at $673 \mathrm{~K}$ at a residual pressure of $5 \times 10^{-9}$ mbar. ${ }^{15}$ Surfaces are characterized by sets of diffracted intensity rods running through bulk Bragg reflections perpendicular to the surface of the crystal. ${ }^{16}$ One can identify two crystal truncation rods originating from the (112) surface and no additional noninteger order rods, consistent with the LEED result of a nonreconstructed surface. This is also exemplified by the $H$ scan shown in Fig. 1(d) intersecting the truncation rod signal at integer $H$ values.

In order to obtain atomic-level information on the structure of the clean $\operatorname{Pd}(112)$ surface, we measured integrated intensities along five crystal truncation rods (in total 118 independent structure factors). The data were collected at $673 \mathrm{~K}$, in order to have a good reference for the subsequent in situ oxidation experiments described in the next section. The experimental magnitudes of the structure factors are presented in Fig. 2. A comparison with the structure factors calculated for a bulk-truncated Pd(112) surface shows that the surface structure deviates significantly from the ideal one (dashed lines in Fig. 2). In general this is not unexpected for vicinal surfaces since significant relaxations of the step atoms may occur, which propagate into the bulk. ${ }^{17}$ For the data presented here, the dominant feature is a reduction of the diffracted intensity in the minima of the CTRs [e.g., at $L=2$ on the $(-1,1)$ rod or at $L=0$ on the $(0,1)$ rod], which is characteristic for a reduced occupancy of the atomic sites in the topmost layer of the (112) surface. ${ }^{18}$

A detailed structural refinement was performed by fitting simultaneously all the measured truncation rods. As a starting model, a $\operatorname{Pd}(112)$ surface was chosen, for which every second surface atomic row was missing, as pictured in Fig. 3(b). Such a 
(a)

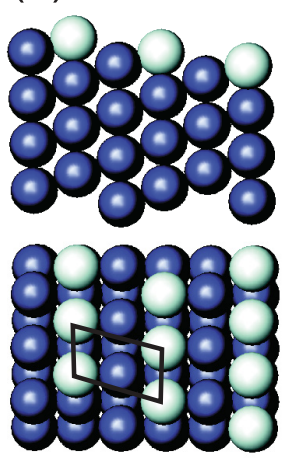

(113)

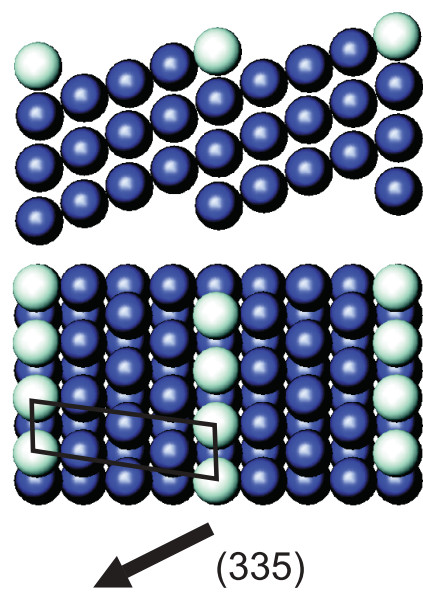

(335)

(b)
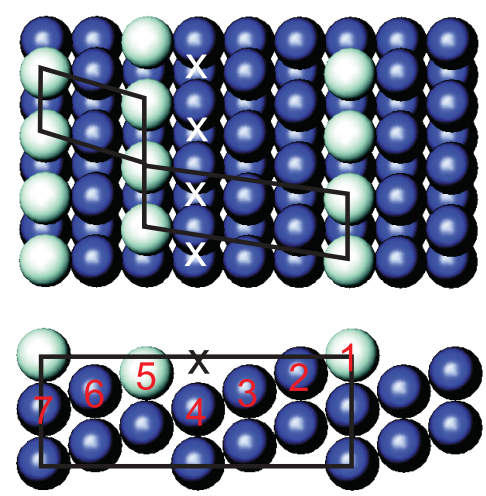

(c)

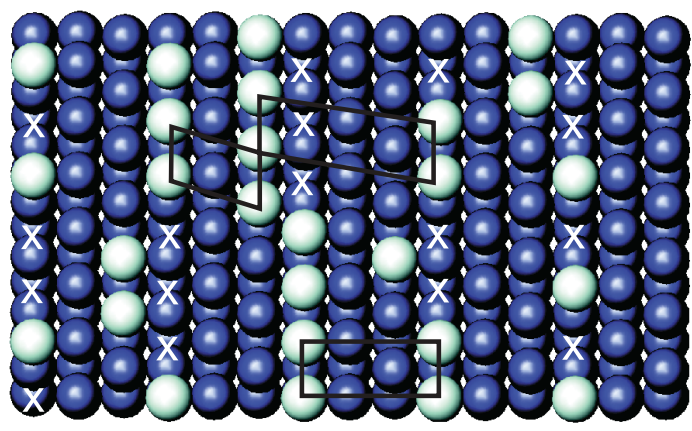

FIG. 3. (Color online) (a) Schematic top and side view of the $\mathrm{Pd}(113)$ and (335) surface. (b) Zipperlike periodic arrangement of (113) and (335) surfaces resulting in a (112) surface with every second atomic row missing along the [010] direction. The (113) and (335) surface unit cells are indicated by solid lines. The cross marks the position of the missing atoms. (c) Realistic model of the defective $\operatorname{Pd}(112)$ surface compatible with the x-ray-diffraction data. The local (113), (335), and (112) geometries are indicated by solid lines.

surface can be considered as a regular, zipperlike arrangement of alternating (113) and (335) facets [see Fig. 3(a)]. During the refinement, a partial occupancy of the vacancy sites of the $\operatorname{Pd}(112)$ surface marked by crosses in Fig. 3(b) was allowed, but the best fit was obtained when keeping these sites unoccupied. For the fit, the $x$ and $z$ positions of the atoms labeled 1-7 in Fig. 3(b) were allowed to relax, which are the atoms sitting at the surface. Displacements of atoms in deeper layers were tested, but they do not improve the fit significantly and are not able to reproduce the observed dips in the structure factors in the minima of the CTRs. The atomic

TABLE I. Fractional atomic coordinates $(x, y, z)$ of the clean $\operatorname{Pd}(112)$ surface with respect to the unit cell marked by the rectangle in Fig. 3(b) ( $a=13.6976 \AA, b=2.7960 \AA$, and $c=4.7931 \AA$ ). The atoms marked 1-7 in Fig. 3(b) are relaxed with respect to their bulk positions (subscript "bulk").

\begin{tabular}{lcccccc}
\hline \hline Atom & $x$ & $x_{\text {bulk }}$ & $y$ & $y_{\text {bulk }}$ & $z$ & $z_{\text {bulk }}$ \\
\hline $\operatorname{Pd}(1)$ & 0.008 & 0.000 & 0.000 & 0.000 & 1.016 & 1.000 \\
$\operatorname{Pd}(2)$ & 0.333 & 0.333 & 0.500 & 0.500 & 0.842 & 0.833 \\
$\operatorname{Pd}(3)$ & 0.833 & 0.833 & 0.500 & 0.500 & 0.841 & 0.833 \\
$\operatorname{Pd}(4)$ & 0.167 & 0.167 & 0.000 & 0.000 & 0.691 & 0.667 \\
$\operatorname{Pd}(5)$ & 0.667 & 0.667 & 0.000 & 0.000 & 0.692 & 0.667 \\
$\operatorname{Pd}(6)$ & 0.000 & 0.000 & 0.500 & 0.500 & 0.500 & 0.500 \\
$\operatorname{Pd}(7)$ & 0.500 & 0.500 & 0.500 & 0.500 & 0.520 & 0.500 \\
\hline \hline
\end{tabular}

positions corresponding to the best-fit model are listed in Table I (residual $\chi^{2}=2.02$ ). The main observation is an outward relaxation of the surface atoms up to $0.08 \AA$ perpendicular to the surface and an in-plane relaxation of atom 1 away from the step edge by $0.11 \AA$.

Since the extended $(2 \times 1)$ unit cell shown in Fig. 3(b) would give rise to reconstruction rods in reciprocal space, which are not observed for the clean surface [see Figs. 1(b)$1(d)$ ], no $(2 \times 1)$ structure with long-range order exists. It is therefore reasonable to assume that the surface is highly defective with a large number of antiphase domain boundaries of the $(2 \times 1)$ superstructure, as illustrated in Fig. 3(c). Note, that the diffraction signal on the crystal truncation rods itself is insensitive to the order of the $(2 \times 1)$ superstructure, since it only probes atomic distances of the unreconstructed surface. Our results demonstrate that already the clean $\operatorname{Pd}(112)$ surface is unstable at elevated temperatures and that it decomposes locally into (113) and (335) patches.

\section{B. Low-pressure regime: Chemisorption on $\operatorname{Pd}(112)$}

In situ oxidation experiments were performed by means of SXRD, as follows: the $\operatorname{Pd}(112)$ crystal was first heated at $673 \mathrm{~K}$ and subsequently exposed to oxygen. The intensity evolution was followed in real time by performing $H$ scans at $K=0$ and $L=1.2$, which is indicated by the white dashed line in Fig. 1(c). The reference scan for the clean surface is shown in Fig. 1(d). Oxygen was applied at progressively higher partial pressures, and whenever changes were observed in the reference scan a characterization of the system was performed by an extended mesh scan.

Already upon exposure to $2 \times 10^{-8}$ mbar oxygen at $673 \mathrm{~K}$, the reference scan changes significantly, as seen in Fig. 4(a), and indicates a doubling of the periodicity in the direction perpendicular to the steps. This observation is in perfect agreement with the LEED data as shown in Fig. 4(b). Strong evidence for step doubling (4.49 $\AA$ ) was obtained also from the STM measurements [see Fig. 4(c)]. The line profile in Fig. 4(d) shows the doubling both of the interstep distance $(2 \times 6.74 \AA)$ and of the step height $(2 \times 2.24 \AA)$, which is necessary in order to maintain the overall orientation of the original (112) surface. These observations could correspond to the formation of either (111) terraces with double steps, i.e., (001) orientation of the microfacets, or to slightly shorter (111) 
(a)

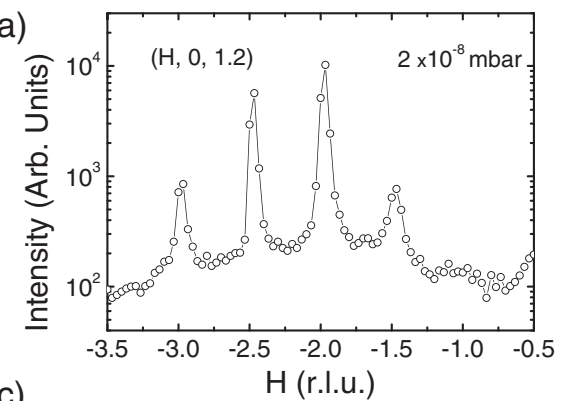

(c)

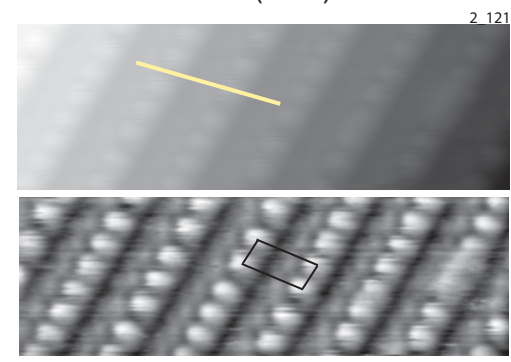

(b)

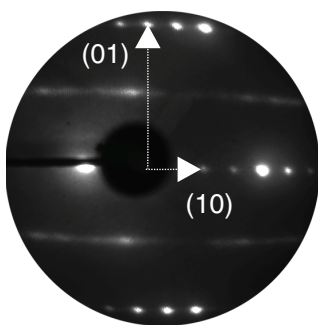

(d)

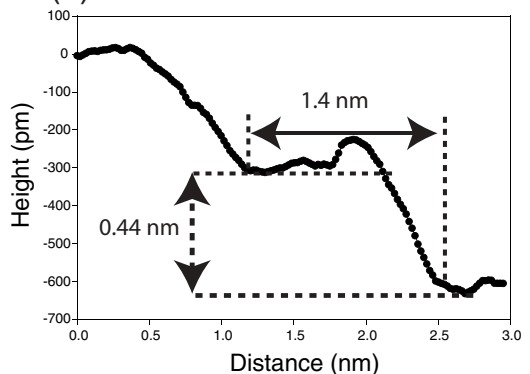

(e)
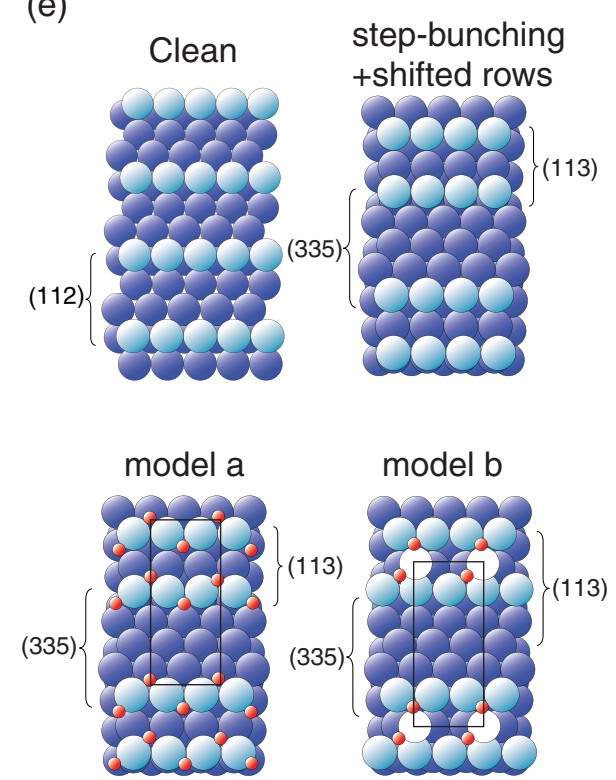

FIG. 4. (Color online) (a) $H$ scan at $(K=0, L=1.2)$ measured at $673 \mathrm{~K}$ and $2 \times 10^{-8}$ mbar oxygen pressure. Half-order satellites indicate a doubling of the periodicity across the steps with long-range order. (b) $(2 \times 2)$ LEED pattern measured at $120 \mathrm{eV}$ after oxidation at $623 \mathrm{~K}$ and $1 \times 10^{-8}$ mbar oxygen. (c) Room temperature STM image $\left(8 \times 4 \mathrm{~nm}^{2}, 0.3 \mathrm{nA}, 2 \mathrm{~V}\right)$ after oxidation at $623 \mathrm{~K}$ and $1 \times 10^{-8} \mathrm{mbar}$ oxygen, where the (111) terraces were made flat (top), and the same image after applying a high-pass filter (bottom). (d) Line profile along the white line marked in (c). (e) The perfect $\mathrm{Pd}(112)$ surface at room temperature (top left), oxygen induced step bunching and shifting of rows (see text) into Pd(113) and Pd(335) steps (top right), and possible oxygen adsorption scenarios leading to a $(2 \times 2)$ reconstruction (see text) (bottom left and right). Oxygen atoms are represented by the small (red) spheres.

terraces as a (335) surface (9.02 $\AA$ ), separated by short (113) terraces $(4.56 \AA)$ yielding a total step-step distance of $13.5 \AA$.

Furthermore, a twofold periodicity along the [010] direction can be seen in Fig. 4(c), which can also be observed as a streaky $(2 \times 2)$ pattern in LEED [Fig. 4(b)]. Therefore an overall oxygen induced $(2 \times 2)$ structure is observed, involving a significant mass transfer of Pd atoms across the surface in line with the high mobility as observed for the clean surface.

The exact surface structure is difficult to provide based on our data, however a few models may be proposed. In the first model, the surface facets change from (112) into (113) and (335) facets, as illustrated in Fig. 4(e). Above, we have demonstrated that the clean surface has a tendency to form (113) and (335) facets at elevated temperatures. Below we show that with increasing oxygen dose the (111) terraces increase further in width and it becomes evident that larger (113) facets are formed on the surface (see Fig. 6). We therefore conclude that the steps in Fig. 4(c) are (113) facets and not double steps with (001) facets. Second, every second step in the (113) surface shifts half a nearest-neighbor distance in the [010] direction [see the unit cell indicated in Fig. 4(c) and the model in Fig. 4(e)]. At this point, we need to add the oxygen atoms, and here the uncertainty is high. A straightforward explanation for the twofold periodicity along the step would be oxygen atoms arranged in a zig-zag pattern below and above the Pd step atoms as observed on other vicinal surfaces at low oxygen coverage, ${ }^{8,19}$ shown in Fig. 4(e) bottom left. The reason for the shifted rows now becomes clear, since without the shifted rows some of the oxygen atoms would have occupied unfavorable bridgelike sites. However, it was recently shown that oxygen may stabilize $\mathrm{Rh}$ adatoms on a

$\operatorname{Rh}(113)$ surface,$^{20}$ and we cannot exclude such a scenario on the $\operatorname{Pd}(113)$ nanofacets. Therefore, we propose a model as shown in Fig. 4(e) bottom right. In this model, the rows are unshifted, and the rectangular unit cell is due to the oxygen stabilized Pd adatoms.

At only slightly increased oxygen pressure $\left(1 \times 10^{-7} \mathrm{mbar}\right)$ and $673 \mathrm{~K}$, the diffracted intensity measured for the reference scan changes again and the reconstruction across the steps is lifted [see Fig. 5(a)]. The CTR signal of the Pd(112) surface is still present, indicating that the surface still exhibits an overall (112) orientation. However, additional intensity appears at $H=-1$, compatible with changes in surface relaxations and/or site occupancies. Additional information comes from the LEED pattern shown in Fig. 5(b): a $(1 \times 9)$ superstructure is observed along the step direction, in line with a strain induced formation of linear oxide chains. ${ }^{8}$

(a)

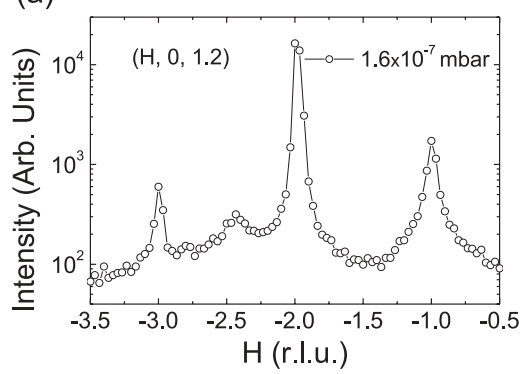

FIG. 5. (a) $H$ scan at $K=0, L=1.2$ measured at $673 \mathrm{~K}$ and $1 \times 10^{-7}$ mbar oxygen. (b) Corresponding LEED pattern showing a $(1 \times 9)$ reconstruction. (b)

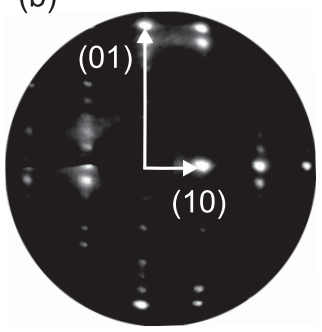




\section{Intermediate-pressure regime: Oxygen chemisorption and faceting}

Figure 6(a) shows the experimental x-ray reciprocal space map of the $(H, 0, L)$ plane measured at $673 \mathrm{~K}$ and $6 \times$ $10^{-7} \mathrm{mbar}_{2}$. The crystal truncation rods are now tilted with respect to the original $L$ direction, and, in addition, they have a different in-plane periodicity as compared to the (112) surface [see Fig. 1(a) for comparison]. This is a clear indication of larger facet formation, since any extended facet having an orientation different than the original surface normal will give rise to truncation rods which are tilted. We have determined the angles between the newly formed facets and the (112) surface to be 5 and $10^{\circ}$, respectively, which correspond well to (335)and (113)-type facets. The LEED image taken after exposure to $6 \times 10^{-7}$ mbar of oxygen at $623 \mathrm{~K}$ is shown in Fig. 6(c). The spots corresponding to the (112) surface disappeared and new spots have emerged, which are much less straightforward to interpret.

The STM image in Fig. 6(d) confirms the in situ X-ray observation and illustrates the presence of the (113) and (335) facets. A schematic representation of these facets is presented in Fig. 6(b). We argue that an increase in the number of the adsorbed oxygen leads to further strain increase in the surface via a local displacement of Pd atoms; this strain is released by step bunching of the (113) and (335) nanofacets into larger terraces. A zoom-in on the (113) terrace is shown in Fig. 6(e), displaying atomic resolution. The distance between the steps or rows is found to be approximately $4.7 \AA$, in good agreement with the expected $4.56 \AA$, while along the steps the distance is measured to be approximately $3 \AA$. The latter value is slightly larger than the expected $2.75 \AA$, possibly due to drift in the image. Surprisingly, the atoms along a line across the steps are not shifted with respect to each step, as would be expected from a (113) surface, indicated by the rectangle in the image. One way to explain this observation is that every second Pd step is shifted half a lattice distance in the [010] direction as was also described in Fig. 4(e). After the rows have been shifted, the oxygen can be added in a similar way as for the $c(2 \times 4)$ structure on $\operatorname{Pd}(110) .{ }^{8,21}$ In fact, the STM appearance is very similar to that of the $c(2 \times 4)$ on $\operatorname{Pd}(110)$. An oxygen induced shifted row has also been observed for the $\mathrm{Pt}_{50} \mathrm{Rh}_{50}(100)$ surface. ${ }^{22}$ We therefore propose a model consisting of a (113) surface with every second row shifted half a lattice distance and the rows decorated as shown in Fig. 6(f). Unshifted rows would have resulted in unfavorable bridgelike oxygen adsorption sites. The structure on the (335)-like facets has not been further evaluated. (a)

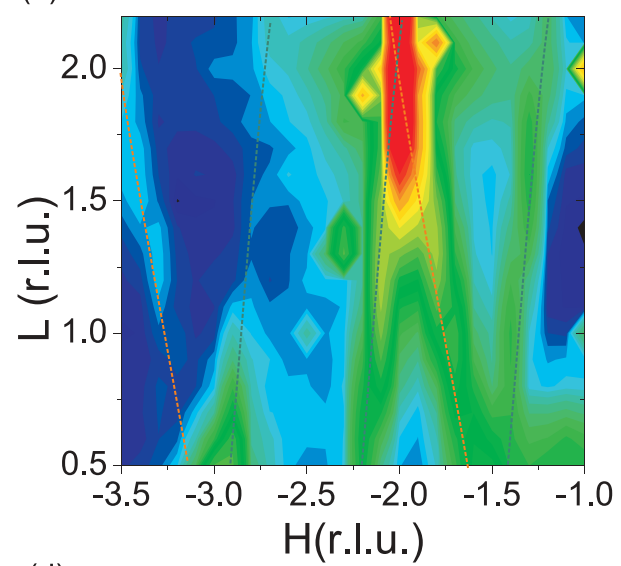

(d)

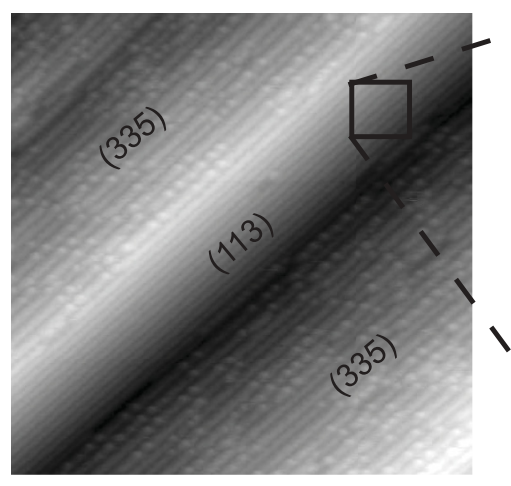

(b)

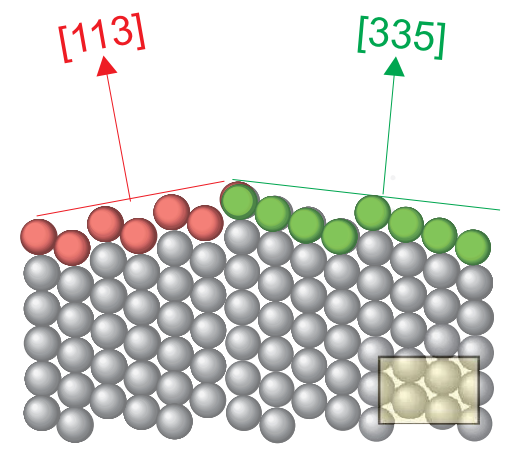

(e)

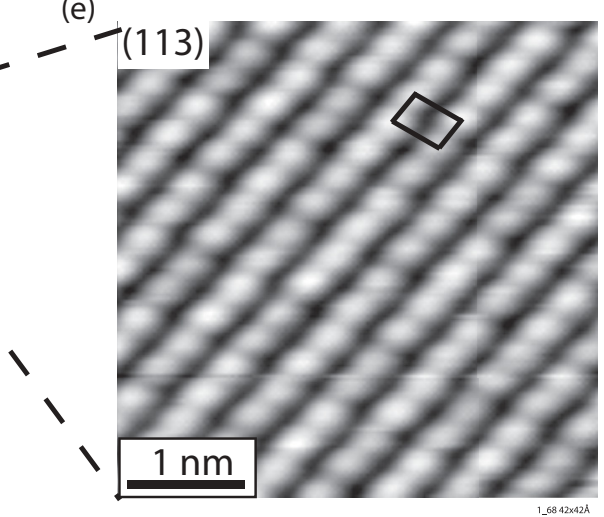

(c)

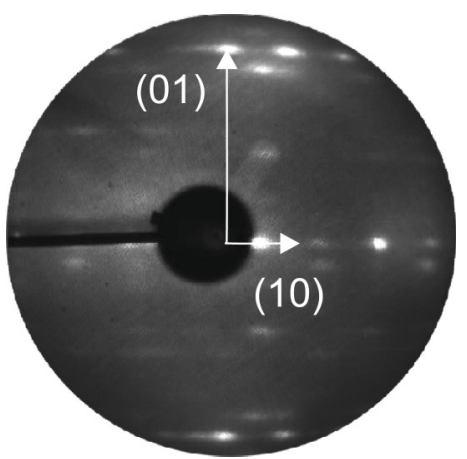

(f)

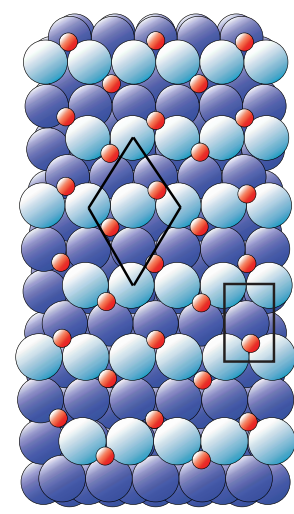

FIG. 6. (Color online) (a) Experimental map of the diffracted intensity in the $(H, 0, L)$ plane measured at $673 \mathrm{~K}^{\text {and }} 6 \times 10^{-7} \mathrm{mbar} \mathrm{O}_{2}$. (b) Schematic representation of the (113) and (335) types of facets (side view). (c) LEED image from the Pd(112) surface after exposure to $6 \times 10^{-7}$ mbar of oxygen at $623 \mathrm{~K}$. The periodicity of the spots both along the $H$ and $K$ directions has changed due to faceting. (d) STM image $(V=0.5 \mathrm{~V}, I=0.1 \mathrm{nA})$ illustrating the presence of the (113) and (335) facets $(40 \times 40 \mathrm{~nm})$. (e) STM image $(V=0.5 \mathrm{~V}, I=0.1 \mathrm{nA})$ from the (113) facets. (f) Model of the surface structure on the (113) facets, indicating the surface unit cell as well as the rectangular shape observed in the STM image in (e). 
(a)

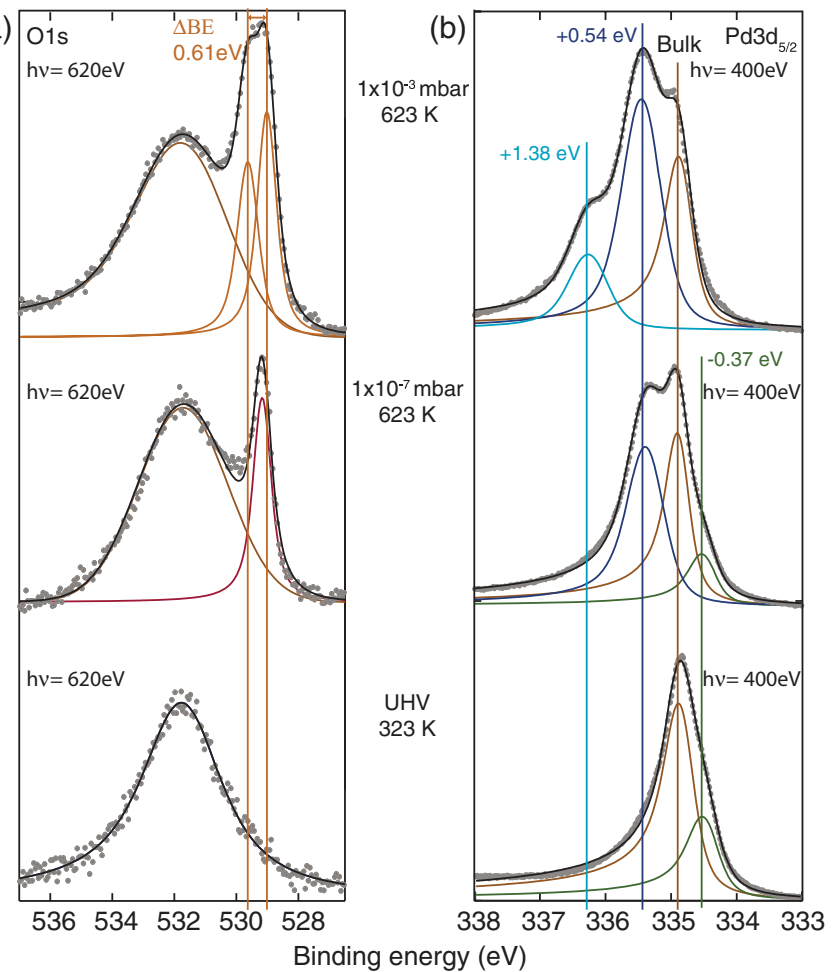

FIG. 7. (Color online) HRCL spectra from the Pd $3 d^{5 / 2}$ and O $1 s$ levels recorded from clean $\operatorname{Pd}(112)$ (bottom) and after exposure at $623 \mathrm{~K}$ and different oxygen pressures: $1 \times 10^{-7} \mathrm{mbar}$ (middle) and $1 \times 10^{-3}$ mbar (top).

Turning to the HRCLS measurements shown in Fig. 7, they provide evidence for the presence of chemisorbed oxygen after oxidation under conditions similar to those described above. The bottom spectra in Fig. 7 shows the O $1 s$ (left) and the $\mathrm{Pd} 3 d^{5 / 2}$ (right) core-level region measured for the clean $\operatorname{Pd}(112)$ surface. As expected, there is no signal from the $\mathrm{O} 1 s$ level. ${ }^{23}$ The decomposition of the clean surface $\mathrm{Pd}$ $3 d$ spectrum reveals the presence of two components: the one on the higher binding-energy side is attributed to the bulk Pd atoms, while the peak on the lower binging-energy side arises from the undercoordinated step atoms, ${ }^{8}$ exhibiting a shift $(-0.37 \mathrm{eV})$ similar to that from the $\operatorname{Pd}(100)$ surface $(-0.35$ $\mathrm{eV}){ }^{24} \mathrm{The}$ Pd atoms at the terrace generate only a small surface core-level shift similar to that from $\operatorname{Pd}(111)^{25}$ and therefore the corresponding signal is hidden under the bulk component.

At on oxygen pressure of $1 \times 10^{-7}$ mbar an oxygen component can clearly be observed in the $\mathrm{O} 1 \mathrm{~s}$ spectra, which appears to be chemisorbed oxygen only. Further, in the $\mathrm{Pd} 3 d$ level the component due to the undercoordinated $\mathrm{Pd}$ atoms has decreased significantly, indicating oxygen induced shifts due to oxygen adsorption at these atoms. In addition, a component due to $\mathrm{Pd}$ atoms coordinated to two oxygen atoms can be observed shifted $+0.54 \mathrm{eV}$ with respect to the bulk component. This value is in line with previously observed twofolded coordinated Pd atoms on a Pd(111) surface and with the zig-zag models as presented above.

After exposure to $1 \times 10^{-3}$ mbar oxygen two components can be observed in the $\mathrm{O} 1 \mathrm{~s}$ level, as well as a $+1.38 \mathrm{eV}$ shifted $\mathrm{Pd}$ component indicating the presence of $\mathrm{Pd}$ atoms coordinated to four oxygen atoms. This is a clear indication on the formation of a surface oxide whose core-level fingerprint resembles that from the surface oxide on $\operatorname{Pd}(111){ }^{25}$

\section{High-pressure regime: Surface oxide formation and refaceting}

A visual inspection of the experimental map of diffracted intensity measured in the $(H, 0, L)$ plane [see Fig. 8(a)] gives evidence that the surface exposed to $673 \mathrm{~K}$ and $1 \times 10^{-4}$ mbar $\mathrm{O}_{2}$ undergoes a second rearrangement: the (113) facet orientation is maintained, while the (335) facets transform into more stable (111) oriented facets. These are schematically shown in Fig. 8(b). In addition intensity rods along $L$ are visible with an in-plane distance of $H=0.25$ evidencing the formation of a $(4 \times 1)$ superstructure across the steps of the original $\operatorname{Pd}(112)$ surface. In the light of our interpretation of the $(2 \times 2)$ superstructure presented above, we argue that this enlarged periodicity might be caused by a regular arrangement of double-sized $\operatorname{Pd}(113)$ and (335) steps, induced by substrate mediated strain interaction. Such a process was also made responsible for the ordering of $\mathrm{Cu}-\mathrm{O}$ chains on the $\mathrm{Cu}(110)$ surface. $^{26}$

The LEED image shown in Fig. 8(c) was taken after similar oxidation conditions. It shows rather diffuse spots, indicative of a less well-ordered surface structure. The STM image in Fig. 8(d) agrees well with the observation from the diffraction studies: the (335) facets have disappeared and instead (111) facets have formed. In addition, the STM measurements revealed that there are regions on the surface covered by an ordered surface oxide structure [see the area indicated (a)

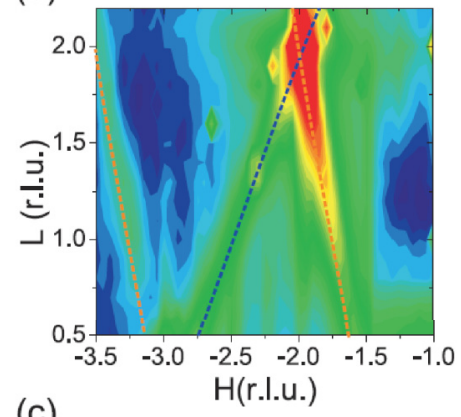

(c)

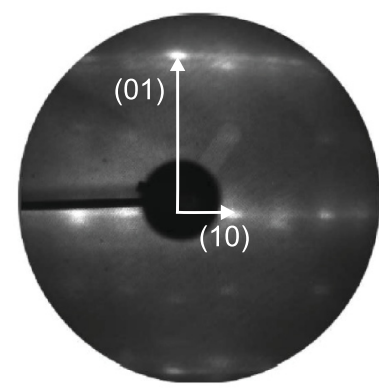

(b)

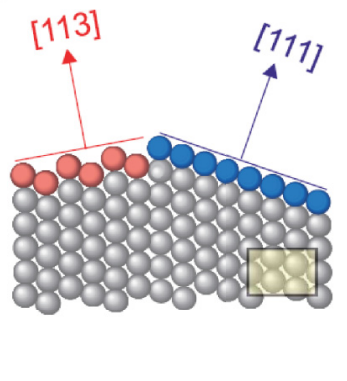

(d)

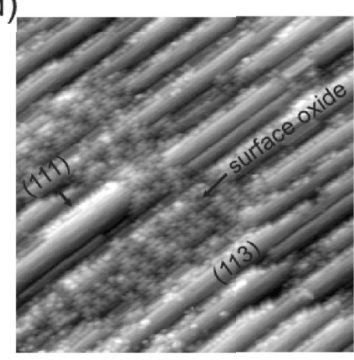

FIG. 8. (Color online) (a) Experimental reciprocal space maps in the $(H, 0, L)$ plane measured at $673 \mathrm{~K}$ and $1 \times 10^{-4}$ mbar $\mathrm{O}_{2}$. (b) Schematic representation of the (113) and (111) types of facets (side view). (c) LEED image from the Pd(112) surface after exposure to $10^{-3}$ mbar of oxygen at $623 \mathrm{~K}$. (d) STM image $(V=0.5 \mathrm{~V}$, $I=0.3 \mathrm{nA}$ ) illustrating the presence of (113) and (111) facets and the formation of the surface oxide $(50 \times 50 \mathrm{~nm})$. 
on Fig. 8(d)]. The structure of the surface oxide observed in our STM measurements resembles closely that of the $\mathrm{Pd}_{8} \mathrm{O}_{8}$ structure that has been reported to form on the $\mathrm{Pd}(111)$ surface upon oxidation at $5 \times 10^{-4}$ mbar $\mathrm{O}_{2}$ at temperatures between 570 and $605 \mathrm{~K}^{27}$ This finding correlates very well with the HRCLS observations: the topmost spectra in Fig. 7 were measured after exposure to $623 \mathrm{~K}$ and $1 \times 10^{-3}$ mbar oxygen. An additional peak appears on the higher binding-energy side in the $\mathrm{Pd} 3 d^{5 / 2}$ region, which indicates the presence of $\mathrm{Pd}$ atoms having a fourfold coordination to oxygen, consistent with the formation of a surface oxide..$^{8,24,25}$ The $\mathrm{O} 1 s$ spectrum is composed of two components, a signature of two different coordinations of the oxygen atoms at the surface, which is in this case a signature of a surface oxide with a O-Pd-O trilayer structure. $^{25}$

\section{E. Near-atmospheric pressures: Bulk oxide formation}

At $673 \mathrm{~K}$, bulk PdO starts to form at an oxygen partial pressure of 0.1 mbar. Figure 9(a) shows an experimental map of the diffracted intensity measured in the $(H, K, 0.1)$ plane after the formation of the bulk $\mathrm{PdO}\left(673 \mathrm{~K}\right.$ and $\left.1 \mathrm{mbar}_{2}\right)$. Bulk PdO with PtS structure exhibits a tetragonal unit cell, and lattice parameters are $a=b=0.303 \mathrm{~nm}$ and $c=0.533 \mathrm{~nm} .^{28}$ In the present experiment, two main orientations have been observed, i.e., (100) and (001) [see Fig. 9(a)]. However, based on the available data, the (101) orientation cannot be excluded, since all the expected in-plane reflections are common with those from the (100) orientation. Additional scans along the $L$ directions (not shown here) were performed in order to probe the out-of-plane structure and orientation of the PdO oxide. No finite thickness oscillations are observed along the $(0,2)$ rod of $\mathrm{PdO}$, indicative of a rather rough oxide surface. From the width of the $(0,2,2)$ PdO Bragg peak, an average thickness of about $5.6 \mathrm{~nm}$ is determined. It has to be mentioned that the observed reflections correspond to epitaxial $\mathrm{PdO}$; no notable formation of powderlike $\mathrm{PdO}$ with random orientation is observed, which would give rise to diffraction rings in reciprocal space.

Apart from the above-mentioned reflections, there are some additional peaks that belong to $\mathrm{PdO}$ and are marked in Fig. 9(a) by the open gray circles. They could not be assigned to any low index $\mathrm{PdO}$ orientation on the $\mathrm{Pd}(112)$ surface and are most likely originating from $\mathrm{PdO}$ formed on the remaining (111) and/or (113) facets.

The above observations describe the typical behavior for bulk oxide growth on $\mathrm{Pd}(112)$ exposed to oxygen at $673 \mathrm{~K}$ and above, which in this temperature range is also observed on low index (100) and (111) Pd surfaces. ${ }^{29,33}$ To test the role of steps upon the kinetic hindrance towards bulk oxide formation, we exposed the $\operatorname{Pd}(112)$ surface at $T=523 \mathrm{~K}$ to oxygen at variable pressure, which is below the bulk oxide formation temperature of low index (100) and (111) surfaces. Figure 9(b) shows the corresponding reference $H$ scans at $K=0$ and $L=$ 1.2. First, changes of the clean surface signal at $H=-3$ are present at $5 \times 10^{-5}$ mbar oxygen pressure, indicating oxygen induced disorder or surface roughening (scan not shown). The signal continues to change significantly only at a pressure of 5 mbars. During the temporal evolution the signal increases first at $H=-2.1$, corresponding to the signal position for
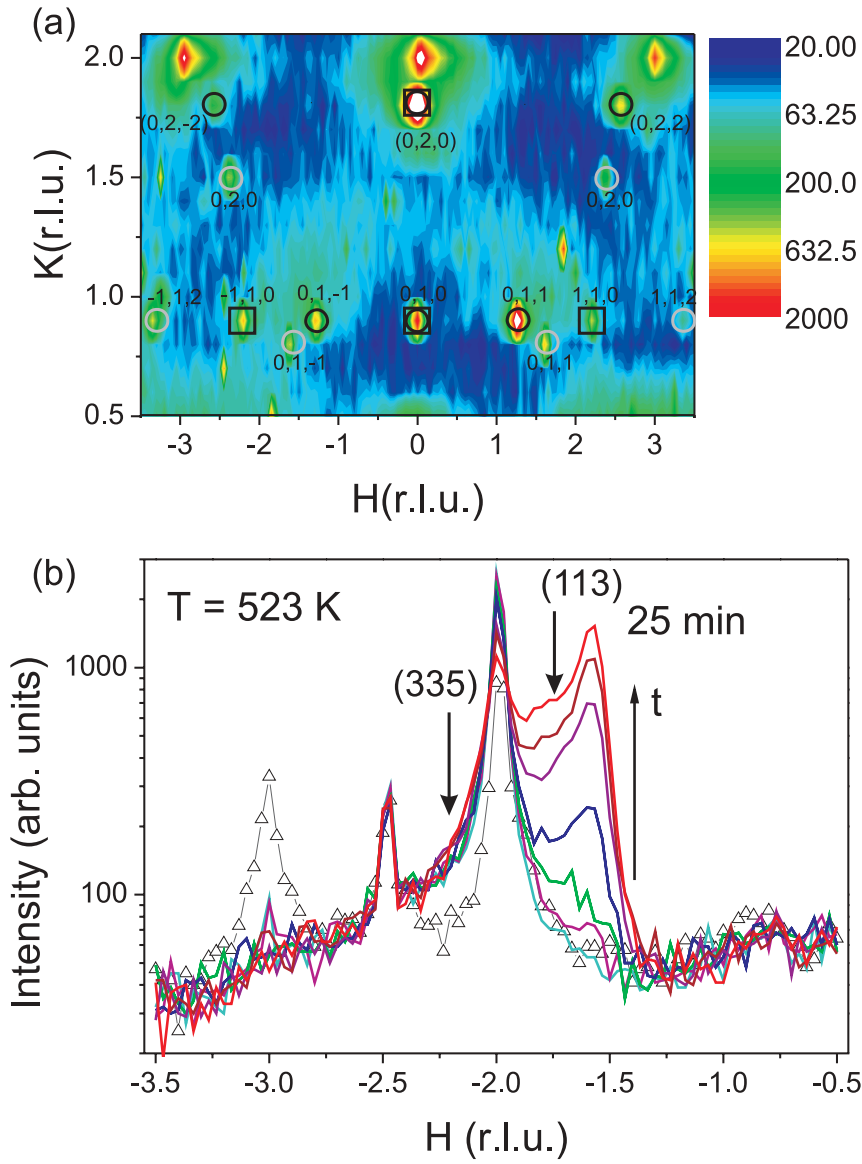

FIG. 9. (Color online) (a) Experimental map of the in-plane diffracted intensity in the $(H, K, 0.1)$ plane measured at $673 \mathrm{~K}$ and 1 mbar $\mathrm{O}_{2}$. Reflections arising from two main orientations of $\mathrm{PdO}$ were observed, i.e., (100) (black open squares) and (001) (black open circles), which are indexed in terms of the $\mathrm{PdO}$ bulk reciprocal lattice. (b) $H$ scan at $K=0, L=1.2$ measured at $523 \mathrm{~K}$ and different oxygen partial pressures from near UHV up to 5 mbars (open triangles, clean surface; solid lines, temporal evolution at 5 mbars). No new peaks appear until the pressure reaches 5 mbars, when the peak evolving at $H=-1.58$ marks the formation of $\mathrm{PdO}$ bulk oxide. In addition, a signal from (113) and (335) facets is observed.

(335) facets. In the later stage the signal at $H=-1.75$ and -1.58 evolves, the first one at the position of (113) facets and the second one at the position of the (110) reflection of the $\mathrm{PdO}$ bulk oxide. As a consequence, the presence of steps at the (112) surface lowers kinetic barriers for bulk oxide formation. The appearance of (335) and (113) facets indicates that under oxygen exposure at $523 \mathrm{~K}$ the surface atoms exhibit already enough mobility to alter the surface morphology on the nanoscale.

\section{DISCUSSION AND CONCLUSIONS}

Table II summarizes the results obtained after oxidation of the $\operatorname{Pd}(112)$ surface at various oxygen pressures together with the values for the relative oxygen chemical potential as defined in Ref. 30. It is apparent that, at intermediate oxygen chemical potentials between -1.6 and $-1.31 \mathrm{eV}$, (113) and (335) facets are stabilized by the chemisorption of 
TABLE II. Overview of the results obtained for the oxidation of the $\mathrm{Pd}(112)$ surface at $673 \mathrm{~K}$. The system behaves similarly at temperatures higher than $523 \mathrm{~K}$.

\begin{tabular}{lcccc}
\hline \hline$T(\mathrm{~K})$ & $p\left(\mathrm{O}_{2}\right)(\mathrm{mbar})$ & $\Delta \mu_{\mathrm{O}}(\mathrm{eV})$ & Surface structure and orientation & Phase \\
\hline 673 & $<1 \times 10^{-10}$ & $<-1.57$ & $(1 \mathrm{x} 1)$ defective $(112)$ & Clean \\
673 & $2 \times 10^{-8}$ & -1.41 & $(2 \times 2)$; periodic $(113)+(335)$ nanofacets & Chemisorbed oxygen \\
673 & $1 \times 10^{-7}$ & -1.37 & $(1 \mathrm{x} 9)$; defective $(112)$ & Chemisorbed oxygen \\
673 & $6 \times 10^{-7}$ & -1.31 & $(113)+(335)$ facets & Chemisorbed oxygen \\
673 & $1 \times 10^{-4}$ & -1.16 & $(113)+(111)$ facets & Surface oxide \\
673 & $1 \times 10^{-1}$ & -1 & $(112)$ defective & Bulk oxide \\
523 & $1 \times 10^{-5}$ & -0.8 & $(113)$ and $(335)$ facets & Chemisorbed oxygen \\
523 & 5 & -0.61 & Bulk oxide \\
\hline \hline
\end{tabular}

oxygen. Already on the clean, nominally (112) oriented surface vacancies are present at $673 \mathrm{~K}$, representing the onset of local (113) and (335) nanofaceting. At $2 \times 10^{-8}$ mbar oxygen pressure the formation of a well-defined, regular structure of alternating (113) and (335) nanofacets is observed. Such gas adsorption induced, regular patterns may form by strain induced step interaction. ${ }^{26}$ At a chemical potential of $-1.31 \mathrm{eV}$ step bunching of the (113) and (335) nanosteps results in the evolution of larger facets (size $>10 \mathrm{~nm}$ ), giving rise to tilted crystal truncation rods in the reciprocal space maps. The formation of (113) facets is in line with theoretical calculations, which predict that at an oxygen chemical potential of $\Delta \mu_{\mathrm{O}}=$ $-1.35 \mathrm{eV}$ the (113) surface energy becomes lower than that of the (112) facets. ${ }^{31}$ This process involves an important mass transport, which is facilitated at elevated temperatures.

A further morphological change takes place at $\Delta \mu_{\mathrm{O}}=$ $-1.16 \mathrm{eV}$, when a transition from (335) to (111) facets is observed, in conjunction with the formation of an ordered surface oxide, acting as a driving force for this process. The (113) faceted areas are surprisingly unaffected by this process, demonstrating their higher stability as compared to the (335) facets. These observations are in contrast to the shape changes of Pd nanoparticles on $\mathrm{MgO}(100)$, for which (112) nanofacets are stabilized under oxygen exposure at $\Delta \mu_{\mathrm{O}}=-1.04 \mathrm{eV}$. We argue therefore that the possibility of strain release may play a very important role for the stability of different facets under gas exposure. Strain relaxation and mass transport processes are facilitated in the case of Pd nanoparticles, for which the (112) faceted area has a size of approximately $1.4 \times 3.5 \mathrm{~nm}$ only. ${ }^{6}$

Finally, at $\Delta \mu_{\mathrm{O}}=-1 \mathrm{eV}$ and $T=673 \mathrm{~K}$ the growth of bulk PdO was observed, close to the theoretical stability limit. $^{31}$ The formation of bulk oxide was also detected at $523 \mathrm{~K}$, indicating a lowering of kinetic barriers for this process as compared to $\operatorname{Pd}(100) .^{32}$ Also at this temperature a rearrangement of the surface predominantly into (113) facets was observed at 5 mbars $\mathrm{O}_{2}$ pressure, giving additional evidence for the high stability of oxygen covered (113) facets.

We conclude that vicinal surfaces represent nice model systems mimicking certain aspects of nanoparticles used for catalytic reactions. Nevertheless, they exhibit their "own life" under oxygen exposure, which alters the strain state of the surface and consequently the stability of the initial vicinal orientation, possibly giving rise to large mass transport and the local formation of differently oriented, new facets. Consequently, an extrapolation to the properties of nanoparticles has to be performed with caution, including also the application of the Wulff construction at the nanoscale.

\section{ACKNOWLEDGMENTS}

Financial support is acknowledged from the European Union under Contract No. NMP3-CT-2003-505670 (NANO2).
*Corresponding author: andreas.stierle@uni-siegen.de

†Present address: Physik-Institute, Universität Zürich-Irchel, CH8057 Zürich, Switzerland.

${ }^{1}$ G. Ertl, H. Knözinger, F. Schüth, and J. Weitkamp, Handbook of Heterogeneous Catalysis (Wiley-VCH, Weinheim, 2008).

${ }^{2}$ E. Lundgren, A. Mikkelsen, J. N. Andersen, G. Kresse, M. Schmid, and P. Varga, J. Phys.: Condens. Matter. 18, 481 (2006).

${ }^{3}$ A. Stierle, N. Kasper, H. Dosch, E. Lundgren, J. Gustafson, A. Mikkelsen, and J. N. Andersen, J. Chem. Phys. 122, 044706 (2005).

${ }^{4}$ G. Zheng and E. Altman, Surf. Sci. 462, 151 (2000).

${ }^{5}$ C. R. Henry, Surf. Sci. Rep. 31, 231 (1998).

${ }^{6}$ P. Nolte, A. Stierle, N. Kasper, N. Y. Jin-Phillipp, N. Jeutter, and H. Dosch, Nano Lett. 11, 4697 (2011).

${ }^{7}$ L. Vattuone, L. Savio, and M. Rocca, Surf. Sci. Rep. 63, 101 (2008).
${ }^{8}$ R. Westerström et al., Phys. Rev. B 76, 155410 (2007).

${ }^{9}$ F. Li, F. Allegretti, F. N. S. Surnev, Y. Zhang, W.-B. Zhang, and K. Reuter, Surf. Sci. 604, 1813 (2010).

${ }^{10}$ A. Stierle, A. Steinhäuser, A. Rühm, F. U. Renner, R. Weigel, N. Kasper, and H. Dosch, Rev. Sci. Instrum. 75, 5302 (2004).

${ }^{11}$ E. Vlieg, J. Appl. Crystallogr. 31, 198 (1998).

${ }^{12}$ E. Vlieg, J. Appl. Crystallogr. 33, 401 (2000).

${ }^{13}$ R. Nyholm, J. Andersen, U. Johansson, B. Jensen, and I. Lindau, Nucl. Instrum. Methods Phys. Res. A 467-468, 520 (2001).

${ }^{14}$ R. Ramsier, K.-W. Lee, and J. T. Yates Jr., Surf. Sci. 322, 243 (1995).

${ }^{15}$ The residual gas is mainly composed of $\mathrm{CO}$ and $\mathrm{H}_{2} \mathrm{O}$, which do not absorb on Pd surfaces under the above-mentioned conditions.

${ }^{16}$ I. K. Robinson and D. J. Tweet, Rep. Prog. Phys. 55, 599 (1992).

${ }^{17}$ P. Müller and A. Saúl, Surf. Sci. Rep. 54, 157 (2004). 
${ }^{18}$ E. Vlieg, A. W. Denier van der Gon, J. F. van der Veen, J. E. Macdonald, and C. Norris, Phys. Rev. Lett. 61, 2241 (1988).

${ }^{19}$ J. Gustafson et al., Phys. Rev. B 74, 035401 (2006).

${ }^{20}$ F. Mittendorfer et al., J. Phys. Chem. Lett. 2, 2747 (2011).

${ }^{21}$ R. Westerström et al., Surf. Sci. 602, 2440 (2008).

${ }^{22}$ M. Sporn, E. Platzgummer, E. Gruber, M. Schmid, W. Hofer, and P. Varga, Surf. Sci. 416, 384 (1998).

${ }^{23}$ The broad peak present in this spectrum is due to the $\mathrm{Pd} 3 p^{3 / 2}$ level which lies at $532 \mathrm{eV}$.

${ }^{24}$ M. Todorova et al., Surf. Sci. 541, 101 (2003).

${ }^{25}$ E. Lundgren, G. Kresse, C. Klein, M. Borg, J. N. Andersen, M. DeSantis, Y. Gauthier, C. Konvicka, M. Schmid, and P. Varga, Phys. Rev. Lett. 88, 246103 (2002).

${ }^{26}$ G. Prevot, B. Croset, Y. Girard, A. Coati, Y. Garreau, M. Hohage, L. Sun, and P. Zeppenfeld, Surf. Sci. 549, 52 (2004).
${ }^{27}$ J. Klikovits, E. Napetschnig, M. Schmid, N. Seriani, O. Dubay, G. Kresse, and P. Varga, Phys. Rev. B 76, 045405 (2007).

${ }^{28}$ J. Waser, H. A. Levy, and S. W. Peterson, Acta Crystallogr. 6, 661 (1953).

${ }^{29}$ E. Lundgren, J. Gustafson, A. Mikkelsen, J. N. Andersen, A. Stierle, H. Dosch, M. Todorova, J. Rogal, K. Reuter, and M. Scheffler, Phys. Rev. Lett. 92, 046101 (2004).

${ }^{30}$ K. Reuter and M. Scheffler, Phys. Rev. B 65, 035406 (2001).

${ }^{31}$ F. Mittendorfer, N. Seriani, O. Dubay, and G. Kresse, Phys. Rev. B 76, 233413 (2007).

${ }^{32}$ E. Lundgren, J. Gustafson, A. Mikkelsen, J. N. Andersen, A. Stierle, H. Dosch, M. Todorova, J. Rogal, K. Reuter, and M. Scheffler, Phys. Rev. Lett. 92, 046101 (2004).

${ }^{33} \mathrm{~A}$. Stierle et al. (unpublished). 